\title{
MEDICAL PROBLEM, CONCEQUENCES TO ORAL CARE IN GERIATRIC PATIENT
}

\author{
Nadia Hardini ${ }^{*}$, Oedijani-Santoso ${ }^{1}$ \\ ${ }^{1}$ Department of Dentistry, Faculty of Medicine, Diponegoro University, Semarang, Indonesia \\ *Corresponding author, E-mail: hardininadia@gmail.com
}

\begin{abstract}
Introduction: Oral health status has a significant impact on the systemic health of elderly patients. Elderly patients without oral diseases are rare to find by dentists. Literature Review: Prevention and treatment for caries and periodontal diseases should be done for elderly patients, except in particular conditions when its not worth the risks treatments. Although the relationship between oral diseases and systemic diseases is still controversial and unresolved, it can be assumed that elderly patients have a greater risk of acute and chronic oral infections due to the use of various drugs and medical disorders, impaired immune function, age related impaired kidney, liver and cardiovascular dysfunction. Conclusion: While on duty, dentists who provides care to elderly patients, must know the medical history and systemic review, and understands general medical problems that affects oral care of the elderly.
\end{abstract}

Keyword : medical problem, elderly, oral treatment

\section{Introduction}

Dentists who treat elderly patients need to know patient's medical history, in order to get sufficient information from the patient and other sources (medical records and families).That informations needed to help dentists give the right oral care treatment according to the patient's needs. Proper medical history requires skill, practice and careful attentions from the dentists. If inaccurate informations are found, contact the patient's medical provider for additional information is highly recommended. ${ }^{1}$

A medical history will help establish the medical risk of various oral treatment procedures, and is necessary to determine additional precautions such as antibiotic prophylaxis, sedation or drug adjustments. Elements of medical history including past history (allergies, medications); systemic review (general, cardiovascular, endocrine, neurology, hematology); family history; socioeconomic history. ${ }^{1,2}$ This article discusses the medical history and systemic review which may be a medical problem and have consequences for the oral care of the elderly.

\section{Common Systemic Conditions in Geriatric Patients}

Degenerative factors and medicaments in geriatric patients can manifest as an oral lesion. In the elderly, patients experience degenerative process (aging), but the quality of life still needs to be maintained, maintaining a healthy body including oral health.,

\section{Allergies}

Allergies are very important factors in Dentistry. Usually, patients do not know their own history of allergies, but the allergic reaction can be life-threatening if not treated immediately (shock anaphylaxis). Dentists are expected to be able to know the treatment for geriatric patients with a history of allergies.

Oral management: the history should be taken if the patient is sensitive to drugs, foods or materials (ex latex) especially to that usually used in dental care (antibiotics, codeine, aspirin). It should also be noted if: 1) there is a family history of asthma and atopy; 2) length of response to injection, ingestion or exposure to the allergen; 3) allergic reactions in the form of: syncope, tachycardia, loss of consciousness, difficulty breathing, or rash; 4) therapies during allergies; and 5) doctor who has treated during allergies. ${ }^{1,3,4}$

\section{Bleeding Disorders}

Bleeding disorders in geriatric patients are variable, usually appears in patients with hematological disorders or patients who are taking a drug. The potential for bleeding disorders determines the risk of oral treatment especially in dental invasive procedures. Bleeding disorders can be caused by inherited or acquired problems. Prolonged bleeding after an 
invasive procedure can be caused by local factors such as local tissue or blood vessel damage or systemic factors. Liver, kidney and bone marrow disease or malignancy will cause coagulopathy and interfere with blood clotting process. ${ }^{5,6}$

Anticoagulant drugs (ex warfarin, aspirin, anti-platelet drugs) will also cause bleeding disorders in invasive dental procedures. Use of herbal supplements (garlic, ginco, ginseng) can cause bleeding disorders, especially when used in combination with anticoagulants. Some bleeding disorders, such as bruises, or petechial rash may be due to coagulopathy or thrombocytopenia. ${ }^{6,7}$

Laboratory tests aim to determine the origin of a systemic condition as a cause of oral bleeding disorders. Laboratory tests including: Prothrombine time (PT), Activated partial thromboplastine time (APTT), Platelet count, Bleeding time (BT), Thrombin time (TT)

Table 1. Laboratory tests to determine Homeostasis ${ }^{5,7}$

\begin{tabular}{ll}
\hline \multicolumn{1}{c}{ Test } & \multicolumn{1}{c}{ Normal Value } \\
\hline Platelet count & $15.000-45.000 / \mathrm{mm}^{3}$ \\
BT & $<7$ minutes \\
PT/INR & $11-13 / 1.0$ \\
TT & $9-13$ \\
\hline
\end{tabular}

Specific coagulopathies that a dentist should study: ${ }^{6,8}$

Hemophilia A (deficiency factor VIII), Hemophilia B (factor XI deficiency), Hemophilia C (factor XI deficiency), and von Willebrand disease are diagnosed with certainty before the individual reaches adulthood. Thrombocytopenia is a common disorder, the platelet count, 100,000 / microL, bleeding usually occurs with a platelet count $<50,000$, and spontaneous bleeding when the platelet count $<20.00$. The appropriate precursor to surgery is hemostatic agent and suturing. If the platelet count $<20,000$ it is necessary to perform a preoperative platelet transfusion and refer to haematologist.

\section{Drugs that caused bleeding: $:^{6,7}$}

1. Aspirin The elderly are often advised by doctors to take aspirin as a prevention against ischemic coronary, but because it has been used for a long time, the elderly sometimes do not consider aspirin as a drug. If an invasive procedure is being performed on a patient, the dentist should postpone the procedure, if it is found that the patient is taking aspirin and nonsteroids or is taking other anticoagulants, to avoid bleeding disorders in the patient. Avoiding bleeding disorders can be accomplished by stopping the aspirin for 5 days prior to the elective surgical procedure.

2. Warfarin (Coumadin) : in patients with atrial fibrillation, atherosclerotic vascular disease, post myocardial infarction (MI), post cerebrovascular accident (CVA), deep venous thrombosis (DVT), warfarin is usually given by a doctor treating coronary heart disease. Oral surgery, scaling, endodontics were performed after PT, APTT and BT tests. The risk of thromboembolic events with discontinuation of warfarin is greater than the risk of oral bleeding from dental procedures.

3. Clopidogrel (Plavix)

Antiplatelet agents are used to prevent atherosclerotic events with a history of MI or CVA. When Plavix is discontinued, normalization of platelet aggregation will only occur 5-7 days after discontinuation of the drug. This medication is generally for patients with coronary stents and should not be stopped for routine dental procedures.

\section{Management of oral care in patients at risk of bleeding ${ }^{5,6,8}$}

-A consult with a doctor treating his systemic condition before any invasive oral treatment procedures.

- Avoiding bleeding can be done with hemostatic measures including primary wound closure. The gelatin that is readily absorbed is inserted into the socket after extraction, then sutured

- soft diet during post extraction will help avoid postoperative bleeding and clot loss

- Prevention of oral disease by routine dental evaluation and control, fluoride use, oral hygiene and diet control should be emphasized to minimize the need for future invasive dental care.

-Written and verbal instructions on postoperative hemostasis are given to the patient and their family.

Cancer

Cancer patients may undergo combination therapy, chemotherapy or radiotherapy. The choice of each therapy is 
considered individually. The patient's overall medical status is a major concern. Important issues for dentists relate to post-radiation and / or chemotherapy treatments.

\section{Radiotherapy}

Radiotherapy for head and neck cancer has a high incidence of oral manifestations. The patient's medical history and treatment plan is very important to prevent oral problems during and after radiation therapy by studying the patient's medical records and frequent discussions with the treating doctor.

Important elements in the medical history of a patient with head and neck Radiotherapy: ${ }^{9}$ 1)When was the cancer diagnosis made; 2) Location and degree histologically; 3) Staging, prognosis, survival rate in $1,5,10$ years; 4) Previous therapy (Radiotherapy, chemo, surgery or combination); 5) Radiotherapy dose; 6) Previous dental history: caries, xerostomia, ability to eat, taste of taste, swallowing, and mouth pain; 7) Risks / benefits of treating the remaining teeth; 8) Osteoradionecrosis if tooth extraction before or during radiation; 9) Anticipating the status of the major salivary glands; 10) Location and level of odontogenic disease and potential for infection (caries, periodontal disease, pericoronitis) during and after radiotherapy

Clinical and radiologic examination of planned / moderate head and neck cancer Radiotherapy: ${ }^{9,10}$

1) The size and proximity of the malignancy to the structure of the oral cavity; 2) Oral soft tissue status and examination of xerostomia, mucositis, mucosal ulcers, exposed bone, bacterial or fungal infections; 3) Trismus or limited jaw mobility; 4) Teeth and periodontium tissue: to determine the number, mobility, caries, sensitivity to percussion, changes in tooth position. This examination is necessary, although some information may overlap, both on panoramic radiographs and on oral clinical examinations.

\section{Oral Complications of Head and Neck Radiotherapy. ${ }^{10-12}$}

A.Mucocitis: Inflammation and thinning oral mucosa

Inflammation is usually short-term but has the potential to be serious and interfere with Radiotherapy schedule and may limit total dosage. Localized / ulcerated mucosal damage is common, leading to an increased risk of bacteremia and secondary infections (eg fungi). Management of mucositis depends on the location, extent, duration, mild or moderate symptoms, given mouthwash with anesthetics for pain, and / or steroids for inflammation. Topical anesthetics can be used with NSAIDs or synthetic opioids. Systemic analgesics / opioids are given when the topical anesthetic activity is short (5-15 minutes)

\section{Topical anesthesia for mucositis pain: ${ }^{9}$}

1)Combination of Maalox or kaolin / pectin and benadryl elixir in a 1: 1 mixture; 2) $2 \%$ combination of concentrated lidocaine or lidocaine / benadryl or lidocaine / maalox; 3) Benadryl kaopectat 50:50; 4) Benzydamine (difflam); 5) Dyclonine; 6) Mouthwash containing steroids, antifungals, antihistamines, and / or topical anesthetics

B. Xerostomia: dry mouth and salivary disfunction

1) dry mouth due to Radiotherapy can be relieved by using a substitute for saliva or drinking water; 2) Pilocarpin hydrochloride (5$7.5 \mathrm{mg}, 3-4$ times / day) or cevimeline $30 \mathrm{mg} 3$ times / day) can increase salivary production; 3 ) Neutral sodium fluoride gargle, fluoride gel or high fluoride toothpaste, used every night after brushing and flossing; 4) Patients require dietary counseling with regular follow up during dry mouth, or indefinitely for patients who always have dry mouth or an increased incidence of caries.

C. Osteoradionecrosis (ORN): alveolar bone exposure clinically visible

ORN occurs in patients with radiation> $5000 \mathrm{cGy}$ to the mandible, causing permanent damage to bone cells and mandibular blood supply. The risk of ORN is not limited to patients with removable dental prostheses or trauma to the mucosa, but also after surgery or other oral infections. Hyperbaric oxygen (HBO) is promoted for therapy but not as a precautionary measure prior to extraction in patients to be irradiated. ${ }^{11}$

D. Loss of taste function: usually occurs in postTR patients, may be exacerbated by the presence of salivary hypofunction, is transient except with high doses of TR. The greatest resolution occurs 3 months post TR but dysgesia can last up to 1 year post TR. It is important to always maintain 
oral hygiene, tongue hygiene and a balanced diet. ${ }^{10}$

E. Infection.

Bacterial infections due to changes in the oral environment during radiotherapy, such as changes in oral flora; loss of protective activity of salivary antimicrobial proteins; systemic immunosuppression; malnutrition due to an increase in refined carbohydrates.

Yeast infections are common during and after Radiotherapy. Clotrimazole troches 4-5 / day at the onset of infection and for the duration of Radiotherapy can clear the infection or reduce its severity. Drinking water can help dissolve the clotrimazole troches in xerostomic patients in order to prolong the contact of the troches with the infected mucosa. ${ }^{3.11}$

\section{When to do oral care in patients with cancer.}

Invasive dental procedures (extraction) are best done before TR or at least 7 days before TR, to give the extraction wound time to heal, so that when TR is performed, the extraction scar has closed. The problem is greater with the mandibular bones than with the maxilla bones. Tooth extraction should be performed atraumatically, with alveoloplasty and primary mucosal closure. An impacted tooth that is not expected to develop caries or periodontitis in the future can be maintained with preventive treatment.

Hyperbaric Oxygen (HBO) before surgery

This therapy is considered for patients who have received high-dose TR and require mandibular extraction, but some literature suggests that regional vascularization with $\mathrm{HBO}$ has shown no improvement. ${ }^{11,12}$

\section{Chemotherapy}

Chemotherapy is treatment of choice for cancer. However, oral complications and oral treatment procedures should always be considered, as they both have a direct or indirect effect on the oral cavity, in particular the ability of the oral mucosa to heal and maintain homeostasis. The most common problems are mucositis, mucosal infections and bacteremia due to damage to the mucosal surface during myelosuppression.

Patient's assesment before chemotherapy. ${ }^{3,9}$ Medical diagnosis: the nature, location and degree of malignancy determine the chemotherapy protocol and the overall prognosis
General status: nutrition, total current blood count (white blood cells and platelets), debilitation, to determine level of tolerance to oral treatment.

Blood count: should be considered when planning an invasive procedure, if the patient has a total white blood cell count $<2000$, absolute PMN <500, platelets <40,000. However, prolonged mucosal / gingival bleeding in routine $\mathrm{OH}$ treatment is unlikely with a platelet count> 25,000

Time to do chemotherapy: if chemotherapy was done the previous month, the oral mucosa and bone marrow may be significantly affected.

Risk of infection and / or bleeding as a result of oral treatment: appropriate oral treatment depends on the problem of teeth and periodontal tissue and patient status, and the consequences of untreated treatment should also be considered. Uncomplicated recovery after an invasive procedure is achieved if the platelet count is maintained> 2000 and the absolute neutrophil count is maintained> 1000 for 7-10 days. It is likely that the patient has a coagulopathy due to his disease (leukemia and poor platelet function), in addition to myelosuppression from his cancer treatment.

\section{Oral Complication of Chemotherapy. ${ }^{10-12}$ a.Prevention of complication}

- The patient should undergo a thorough clinical and radiologic oral examination

- Tooth extraction that cannot be maintained due to periodontal disease or caries

- Comprehensive oral prophylaxis and oral hygiene education. Educate the patient about the relationship between odontogenic disease and oral problems during and after chemotherapy

- Oral antifungal prophylaxis (miconazole or fluconazole) can be given especially for patients with neutropenia

- Neutral sodium fluoride gargle or fluoride gel to prevent dental caries

- Timing of oral treatments such as brushing / flossing, topical anesthetics and antifungals is important so that it does not diminish the benefits of each treatment.

b. Jaw osteonecrosis: possibly in alveolar bone as a result of exposure to radiotherapy, found since 30 years ago. The prevention of oral complications has resulted in a significant reduction in incidence, but patients who have 
undergone radiotherapy with tumoricidal doses of the jaw are at risk of lifelong osteonecrosis. Currently the number of reports of osteonecrosis from bisphosphonate drugs is increasing ${ }^{9,10}$

c. Infection: periodontal abscesses can occur after chemotherapy and the infection must be managed promptly. Antibiotics are less effective for myelosuppression, so it is important to consult with the medical oncologist treating the patient to choose the right antibiotic. During neutropenia (absolute neutrophil count $<500$ microliters), broad-spectrum parenteral antibiotics are required to treat gram-negative and aerobic bacteria. Elective dental treatment should wait until the absolute neutrophil count has risen to> 1000 and the platelets to> 50,000. 10,12

d. Bleeding: usually occurs in the gingival cleft, because the platelet count is $<20,000$. Bleeding is treated by pressing the gauze soaked in salt, with moderate pressure, for 2-3 minutes, if it fails to stop the bleeding, it is replaced with gauze soaked in thrombin, applied locally to the area, and held for several minutes. The gauze is gently removed so as not to disturb the newly formed blood clot. Avoid gingival manipulation (chewing or brushing teeth) 48-72 hours after oral bleeding or until the platelet count is significant. ${ }^{11}$

e. Pain due to mucositis: widely used topical anesthetics to relieve this complaint, can be started with one topical anesthetic and replaced by another if ineffective. Systemic opioids may be given if topical anesthetics are inadequate or the lesion is extensive. Localized areas of mucosal pain can be managed with topical Benzocaine in orabase or in combination with other anesthetic or anti-inflammatory preparations. $^{9}$

f. Nutrition. weight loss being a short or medium term side effect due to mouth or throat pain, nausea / vomiting, poor appetite or diarrhea. Consultation with a dietitian is indicated to find foods that are well tolerated and maintain adequate nutrition. Oral cavities that are sore, dry, or ulcerated, are given a soft solution (soda and water) to keep the mucosa moist and clean. A soft and / or liquid diet including ice cream or melon juice can be consumed. Avoid acidic or spicy foods (orange juice and fruit, herbs and spices), alcohol, cigarettes, and hot or very cold foods. Sugar free candy or mint can stimulate the production of saliva, prefer soft candy and not sharp, because it can injure the oral mucosa. ${ }^{12}$

\section{Cardiovascular disease}

In general, sometimes the patient looks good/healthy, but turns out to have cardiovascular problems that need to be considered before oral procedures, especially invasive procedures. After obtaining a medical history, it is necessary to consult a cardiologist who treats the patient by explaining in detail the possible treatments/actions to be performed. Heart conditions that need to be considered by dentists because they are a high risk of endocarditis in oral procedures (AHA, 2007) ${ }^{6,7}$ as if 1) prosthetic heart valve; 2) Previous infective endocarditis; 3) congenital heart disease (CHD); 4) Heart transplant

All oral procedures with manipulation of the gingival tissue or the periapical area of the tooth or perforation of the oral mucosa, should be given prophylactic antibiotics prior to the procedure.

Tabel 2. Oral Profilaksis (AHA, 2007) ${ }^{7}$ Single Dose 30-60 minutes before oral treatment ${ }^{7}$

Oral: Amoksisilin; adult 2g; children $50 \mathrm{mg} / \mathrm{kg}$

Or i.m or iv, same dose with per oral

Alergic to Penisilin, clindamycin; adult $600 \mathrm{mg}$, children $20 \mathrm{mg} / \mathrm{kg}$

Or, i.m or i.v, same dose with per oral

\section{Assessment of the risk of bacteremia in oral procedures}

The oral cavity has a high bacterial population, so bacteremia can occur as a result of periodontal tissue manipulation. Some literature states that invasive oral procedures are a cause of bacteremia in cardiac infections.

Myocardial infarction (IM): the main concern is the prevention of additional infarction and damage to the heart muscle. It is advisable for patients to delay elective care for the conventional period 6 months after IM. Consult with the treating doctor for additional medical history prior to invasive procedures as patients usually use warfarin or aspirin therapy for anticoagulants. Use caution using epinephrine as a vasoconstrictor in local anesthesia and consider nitric oxide sedation. Postoperative control is important. 13-16 
Table 3. Blood Pressure Categories ${ }^{7,8,17}$

\begin{tabular}{lccc}
\hline & Systolic $(\mathbf{m m H g})$ & & Diastolic (mmHg) \\
\hline Normal & $<120$ & and & $<90$ \\
Elevated & $120-139$ & or & $88-89$ \\
Hipertension & & & \\
$\begin{array}{l}\text { stage 1 } \\
\text { Hipertension }\end{array}$ & $140-150$ & or & $90-99$ \\
stage 2 & $>150$ & or & $>100$ \\
\hline
\end{tabular}

Elective treatment should be avoided if the systolic blood pressure is $>180 \mathrm{mmHg}$ or diastolic > $100 \mathrm{mmHg}$. Hypertension often leads to increased blood loss during surgery. Monitor blood pressure before, during and after treatment. Patients may experience a drop in blood pressure after local anesthesia. Patients should also be monitored for drug-related xerostomia and gingival enlargement.

\section{Other heart considerations}

Cardiac drug interactions: should be careful using respiratory depressants such as opioids, barbiturates and other sedatives that can worsen cardiovascular status. Nitrogen oxide/oxygen can be used safely in cardiac patients, but oxygen content should not fall below $25 \%$. The use of local anesthetics with vasoconstrictors is controversial. The benefit of vasoconstrictor is that the anesthesia is deeper and prolongs the effect of anesthesia, but the risk may be greater in most cases. Avoid epinephrine concentrations> 1: 100,000 . It is important to give the injection slowly (after aspiration), monitor the increase in blood pressure and heart rate and wait for 2 minutes. ${ }^{18-20}$

\section{Stroke}

Stroke is paralysis or weakness of the muscles including the facial muscles, with initial symptoms of headache, nausea and vomiting as well as numbness or paralysis on one side of the body. Caused by 3 events: 1) small or large vessel thrombus, usually in patients with hypertension and DM. Transient ischemic attack (TIA) may be an early sign; 2) embolus, which usually originates from the heart or neck, or atrial fibrillation. Occurs suddenly and improves slowly; and 3) bleeding that is usually spontaneous, or secondary to hypertension or aneurysms, sometimes due to coagulopathy, onset of symptoms suddenly. ${ }^{21}$

\section{Oral Care Management:}

Stroke patients may be taking anticoagulants such as warfarin, so they are evaluated for possible bleeding risks with the PT, BT and APTT tests when an invasive dental procedure is to be performed. One of the main concerns for stroke patients is their ability to maintain proper oral hygiene due to motor and cognitive problems. In such cases, the caregiver of the patient is responsible for the prevention of oral diseases. ${ }^{3.21}$

\section{Endocrine Imbalance}

Diabetes Mellitus (DM): metabolic disorders caused by injury to insulin secretion or tissue sensitivity, with the onset of hyperglycemia. People with DM 20\% are> 65 years old. Patients with persistent hyperglycemia may not be aware of their condition, resulting in delayed diagnosis and a greater chance for other organ abnormalities. In the history, it is necessary to ask about the length of suffering from DM, antiDM drugs, therapy and complications obtained, as well as the treating doctor. ${ }^{22}$

Symptoms: polidipsi, nocturia, malaise, decreased appetite which causes nausea, vomiting, hyperapnuea and coma (diabetic acidosis).

\section{Oral care management ${ }^{22-24}$}

The main concern is preventing hypoglycemia and the possible response to tooth and periodontal tissue infections in uncontrolled DM. Although it is generally felt that all DM patients have an increased risk of oral infections and impaired wound healing, this does not need to happen except for uncontrolled DM patients with insulin dependence.

Controlled Diabetics: Morning is the best time to find out sugar levels because at that time blood sugar levels are high enough so the risk of hypoglycemia is low. Prior to the treatment procedure, it is confirmed that the patient has had breakfast, and is drinking orange or other fruit juices so that he is expected to have enough blood glucose during the oral treatment. Prophylactic antibiotics are rarely given for surgical procedures because there is no scientific data to support this procedure. Although in vivo animal studies have shown that surgical procedures increase the risk of infection due to decreased neutrophil function, they are not clinically proven. 
Uncontrolled Diabetics: consultation of the attending physician is required to consider whether the patient should be admitted to a planned restorative procedure or extensive invasive surgery. Avoid procedures that are stressful and that slow down healing and the insulin dose may need to be adjusted before and after treatment to improve glycemic control. Xerostomia and dehydration are common in DM and predispose to oral disease in patients and interfere with retention of loose dentures. There are changes in the oral mucosa and possible changes in the ecology of oral bacteria, such as lichen planus, fungal infection, hot and dry mouth. It is important to know the general comorbidities of hypertension, obesity, lipid disorders and smoking because they play a role in the development of cardiovascular disease.

\section{Thyroid disorders}

The thyroid hormones, thyroxine (T4) and triiodotiromin (T3) affect metabolic processes, tissue growth, and energy metabolism. Thyroid hormone excess or deficiency if not managed properly can lead to serious medical complications. Thyrotoxic can occur due to stress, trauma, or acute infection, especially if the patient is not well controlled medication. Symptoms of thyrotoxicosis include fever, nervousness, restlessness, altered mental status, sweating, tachycardia, heat intolerance, weight loss, nausea and vomiting, hypertension and exophthalmus. Weight loss and decreased appetite in general are common with the aging process. $^{3}$

Oral care management of Hypothyroidism:

Important elements that should be explored in the patient's history include: 1) the causes of decreased hormone levels; 2) levels of hypothyroid hormone; 3 ) current therapy; and 4) the symptoms that arise.

Oral care management of Hyperthyroidism: the patient may experience anorexia and heart conditions such as atrial fibrillation and congestive heart failure. If possible, use antibiotics / analgesics for acute oral infections until the thyroid is under control. An excessive increase in thyroid hormone will cause osteoporosis to occur more rapidly, it appears on the radiograph.

Although the neutrophil count in hyperthyroid patients is decreased, this is unlikely to have an impact on oral infections. Treatment should be delayed if the patient is untreated or uncontrolled or presents with hyperthyroid symptoms (tachycardia, hypertension). It is important to carry out oral care procedures with care so as not to cause stress, as well as to prevent infection. Managing hyperthyroid patients does not require adjustments to oral management, whereas for hypothyroid patients, dentists should be aware of the patient's susceptibility to cardiovascular disease. Patients with good control have no contraindications for oral treatment. ${ }^{24-26}$

\section{Liver disease}

The number of deaths from liver disease increases rapidly after loss of liver volume and impaired liver cell function and regeneration. The consequence in the elderly is an increase in liver disease and poor and inefficient drug metabolism. Other disorders are hepatitis A, B, C, D. ${ }^{7,27}$

\section{Oral Care Management}

1). Must have a thorough medical history, especially current liver function and complications of cirrhosis (ascites, hypertension, coagulopathy); 2). Avoid elective treatment in patients with active hepatitis; 3 ). Evaluate liver damage before prescribing certain drugs (diazepam, paracetamol) because drug consumption will make the situation worse, as well as for some local anesthetics, analgesics, sedatives and antibiotics. Before any invasive action is carried out, the factors involved in homeostasis must be examined, for example the PT, BT test, and consult with the treating doctor. $^{27-29}$

\section{Kidney disorders}

Aging increases the likelihood of chronic kidney failure (CRF). It is important to understand of the patient's current kidney status to avoid complications with oral treatments and invasive drugs. The incidence of CRF increases due to the increasing number of people with DM. Loss of kidney function results in a variety of problems for dental patients: drug elimination, uremia and cardiovascular complications such as hypertension. ${ }^{30-32}$ 


\section{Oral care management}

Hemodialysis requires the patient to have access (shunt) to the circulation, so blood pressure cannot be measured in the shunt-attached arm. Dentists should be familiar with the doses of antibiotics and antifungals in patients with renal failure. The initial dose of an antifungal or antibiotic must be the same as that of a patient with normal kidney function to achieve a therapeutic blood level. The maintenance dose should be reduced so that the concentration in the blood does not continue to increase before the next scheduled dialysis, at which time all medication is likely to be eliminated. For example, potassium-containing penicillin can be given for short-term use (several days) but not for long-term, because the potassium level is already high in the absence of dialysis. Erythromycin is used as an alternative medicine. Aminoglycosides, tetracyclines and cephalosporins should be avoided because of nephrotoxicity. ${ }^{32-35}$ Heparin is used during dialysis, so bleeding may occur during oral procedures given the short half-life of heparin. The patient is usually tired after dialysis and may not tolerate the current oral treatment, so the oral treatment is carried out the day after dialysis. Prophylactic antibiotics for dialysis patients undergoing oral procedures are urgently needed because they are useful in preventing infection in dialysis shunts. Kidney transplant patients use immunosuppressive drugs for life. The issue of the need for antibiotics and / or steroid prophylaxis for dental procedures is controversial. Watch for enlargement of the gingiva if the patient is taking cyclosporine. Oral disease and infections should be treated aggressively to avoid possible acute exacerbations and complicate the medical management of immunosuppression. ${ }^{35-37}$

\section{Pulmonal Disease}

Asthma i.e. episodic syndrome is an acute or subacute narrowing of the airway or bronchospasm that is reversible. Narrowing of smooth muscle, with edema of the bronchial mucosa and mucus formation, predisposes to infection. Stress is a trigger for attack, as well as cold air, pollutants, respiratory infections, exercise, aspirin, medical non-compliance. Several dental materials have been reported to trigger asthma attacks, including toothpaste, sealants, tooth enamel dust, methyl methacrylate. Severe asthma is treated with steroids.

When taking steroids, one should be aware of the possibility of adrenocortical insufficiency and oral candidiasis. Theophyllin or aminiphyllin, erythromycin and clindamycin can increase theophyline levels and produce theophylin toxicity. It is important to know that about $5 \%$ of asthma patients have aspirin sensitivity. 3,38

\section{Oral care management}

It is important to prevent asthma attacks in a dental clinic. The medical history should include attack frequency, triggers for asthma attacks, and frequency of visits to the emergency room. Elective dental treatment is performed in well controlled patients. Prevent asthma attacks by avoiding oral treatments during respiratory infections and avoiding aspirin as well as drugs containing NSAIDs, epinephrine (controversial) and antihistamines.

Patients are asked to bring anti-asthma medication with each oral treatment procedure. To relieve stress, each treatment procedure is brief. Sedation can be given to minimize the risk of asthma attacks.39,40 Several studies have shown that asthma predisposes to an increased incidence of caries due to xerostomia effects of B2 agonists, calculus, gingivitis, periodontal disease. Asthma patients are encouraged to gargle water after using the inhaler. In the event of an acute asthma attack, stop oral care procedures, remove all intraoral devices, place the patient in a relaxed position, ensure that the airway is open and administer oxygen. If it improves, give subcutaneous epinephrine (1: $1000,0.01 \mathrm{mg} / \mathrm{kg}) .{ }^{40}$

\section{Conclusion:}

It has been explained that the various health statuses of the elderly that must be considered by dentists who carry out oral care, need to be observed through history, patient complaints, history of illness and systemic disorders, drugs consumed, doctors who treat them and the results of existing supporting examinations such as laboratories, Ro photos and others. Dentists should know the oral manifestations and possible complications of diseases and disorders experienced by patients during oral treatment, the examinations that must be carried out, as 
well as how to handle and prevent them as well as educate the patient and his family.

\section{References:}

1. Lockhart PB. Oral Medicine and Medically Complex Patients. $6^{\text {th }}$ ed. 2013. WileyBlackwell, Ames, IA

2. Meurman JH, Hamalainen P. Oral health and morbidity-implications of oral infections on the elderly. Gerodontology. 2006; 23(1): 3-16

3. Lockhart PB, Furney S. Medical issues in the dental care of older adults. In: Textbook of Geriatric Dentistry. $3^{\text {rd }}$ ed. PoulHolmPerdersen, Walls AWG, Ship JA editors. Wiley Blackwell. 2015: 81-100

4. Ismail IH, Tang ML. Oral immunotherapy for the treatment of food allergy. Isr Med Assoc J. 2012;14(1):63-9.

5. Brennan MT, Shariff G, Kent ML, Fox PC, Lockhart PB. Relationship between bleeding time test and postextraction bleeding in a healthy control population. Oral Surgery, Oral Medicine, Oral Pathology, Oral Radiology and Endodontology. 2002; 94(4): 439-43

6. Grines CL, Bonow RO, Casey DE Jr, Gardner TJ, Lockhart PB, Moliterno DJ et al. Prevention of premature discontinuation of dual antiplatelet therapy in patient with coronary artery stents: a science advisory from the Americam Heart Association, American College of Cardiology, Society for Cardiovascular Angiography and Intervention, American College of Surgeon, and American Dental Association, with representation from the American College of Physicians. Journal of the American Dental Association. 2007; 138(5): 652-5

7. Lockhart PB, Gibson J, Pond SH, Leitch J. Dental management considerations for the patient with an acquired coagulopathy. Part 1: Coagulopathies from systemic disease. British Dental Journal. 2003; 195(8): 43945

8. Patton LL. Bleeding and clotting disorders. In: Burket's Oral Medicine. Greenberg, Glick, Ship editors. 11th ed. 2008. BC Decker Inc, Hamilton. 412-22

9. Rubenstein EB, Peterson DE, Schubert M, Keefe D, Mc Guire D, Epstein J, et al. Clinical practice guidelines for the prevention and treatment of cancer therapyinduced oral and gastrointestinal mucisitis. Cancer. 2004; 100(s9): 2026-46

10. Epstein J, Van der Waal I. Oral Cancer. In: Burket's Oral Medicine. Greenberg, Glick, Ship editors. 11th ed. 2008. BC Decker Inc, Hamilton. 177-85

11. PattonLL. Hematologic disease. In: Burket's Oral Medicine. Greenberg, Glick, Ship editors. 11th ed. 2008. BC Decker Inc, Hamilton. 409-10

12. Sonis ST, Elting LS, Keefe D, Peterson DE, Schubert M, Hauer-Jensen $M$, et al. Perspectiveson cancer therapy-induce mucosal injury: pathogenesis, measurement, epidemiology, and concequences for patients. Cancer. 2004; 100(S9): 1995-2025

13. Bavitz JB. Dental menegement of patients with hypertention. Dental Clinic of North America. 2006; 50(4): 547-62

14. Chobanian AV, Bakris GL, Black HR, Cushman WC, Green LA, Izzo JL Jr, et al. The seventh report of the joint national committee on prevention, detection, evaluation, and treatment of high blood pressure. JAMA. 2003; 289(19): 2560-72

15. Lockhart PB, LittmannL, Glick $M$. Deseases of the cardiovascular system. In: Burket's Oral Medicine Diagnosis and Treatment. Greenberg M, Glick M, Ship JA editors. BC Decker Inc. 2007: 323-40

16. Lockhart PB, Loven B, Brennan MT, Fox PC. The evidence base for the efficacy of antibiotic prophylaxis in dental practice. Journal of American Dental Association. 2007; 138(4): 458-74

17. Robert HW, Mitnitsky EF. Cardiac risk stratification for posmyocardial infarction dental patients. Oral Surgery, Oral Medicine, Oral Pathology, Oral Radiology and Endodontology. 2001; 91(6): 676-81

18. Kamath MM, Mala K, Thomas MS. Modification of Dental Care for Patients with Cardiac Disease. OHDM. 2016;15(5): 286-90

19. Swantika Chaudhry, Ritika Jaiswal, Surender Sachdevab. Dental considerations in cardiovascular patients: A practical perspective. Indian Heart J. 2016; 68(4): 572-575.

20. Lockhart PB, Littman L, Glick M. Diseases of cardiovascular system. In: Burket's Oral 
Medicine. Greenberg, Glick, Ship editors. 11th ed. 2008. BC Decker Inc, Hamilton. $323-40$

21. Rose LF, Mealey B, Minsk L, Cohen DW. Oral care for patients with cardiovascular disease and stroke. JADA. 2011; 133(Suppl 1): $37 \mathrm{~S}-44 \mathrm{~S}$

22. Mc Kenna SJ. Dental management of patients with diabetes. Dental Clinic of North America. 2006; 50(4): 591-606

23. Anthony TV. Dental considerations for the treatment of patients with diabetes mellitus. JADA. 2003; 134: 24S-32S

24. Akintoye SO, Collins MT, Ship JA. Diabetes Mellitus and endocrine diseases. In: Burket's Oral Medicine. Greenberg, Glick, Ship editors. 11th ed. 2008. BC Decker Inc, Hamilton. 509-20, 527-30

25. Little JW. Thyroid disorders Part I: hyperthyroidism. Oral Surgery, Oral Medicine, Oral Pathology, Oral Radiology and Endodontology. 2006;101(3): 276-84

26. Pinto A, Glick M. Management of patients with Thyroid disease: oral health considerations. Journal of American Dental Association. 2002;133(7): 849-58

27. Firriolo FJ. Dental management of patients with end-stage liver disease. Dental Clinic of North America. 2006; 50(4): 563-90

28. Schmucker DL. Age-related changes in liver structure and function: Implication for disease? Experiment Gerontology. 2005; 40(8-9): 650-9

29. Marta Cruz-Pamplona, María MargaixMuñoz, Maria Gracia Sarrión-Pérez. Dental treatment in patients with liver disease. J Clin Exp Dent. 2011; 3(2):e127-34.

30. Kerr AR. Update on renal diseasefor the dental practitioner. Oral Surgery, Oral Medicine. Oral Pathology, Oral Radiology and Endodontology. 2001; 92(1): 9-16

31. Fulvia Costantinides, Gaetano Castronovo, Erica Vettori, Costanza Frattini, Mary Louise Artero, Lorenzo Bevilacqua, Federico Berton, Vanessa Nicolin, Roberto Di Lenarda. Dental Care for Patients with End-Stage Renal Disease and Undergoing Hemodialysis. Intr. Dent. J. 2018; 2018: 9610892.

32. De Rossi SS, Cohen DL. Renal disease. In: Burket's Oral Medicine. Greenberg, Glick,
Ship editors. 11th ed. 2008. BC Decker Inc, Hamilton. 379-83

33. Renal Palmer S. C., Ruospo M., Wong G., et al. Dental health and mortality in people with end-stage kidney disease treated with hemodialysis: a multinational cohort study. American Journal of Kidney Diseases. 2015; 66: 666-676.

34. Guggenheimer J, Eghtesad B, Stock DJ. Dental management of the (solid) organ transplant patient. Oral Surgery, Oral Medicine, Oral Pathology, Oral Radiology, and Endodontology. 2003; 95(4): 383-389. doi: 10.1067/moe.2003.150.

35. Georgakopoulou EA, Achtari MD, Afentoulide N. Dental management of patients before and after renal transplantation. Stomatologija. 2011; 13:107-112.

36. Vasanthan A, Dallal N. Periodontal treatment considerations for cell transplant and organ transplant patients. Periodontol 2000. 2007; 44: 82-102.

37. Martí Alamo S, Gavaldá Esteve C, Sarrión Pérez MG. Dental considerations for the patient with renal disease. Journal of Clinical and Experimental Dentistry. 2011; 3: E112-E119. doi: 10.4317/jced.3.e112.

38. Steinbacher DM, Glick M. The dental patient with asthma. An update and oral health considerations. Journal of the American Dental Association. 2001; 132(9): 1229-39

39. Desai S, Scannpieco FA, Lepore M, Analik $\mathrm{R}$, Glick M. Disease of respiratory tract. In: Burket's Oral Medicine. Greenberg, Glick, Ship editors. $11^{\text {th }}$ ed. 2008. BC Decker Inc, Hamilton. 311-13

40. Ariadna Claramunt Lozano, Gracia Sarrión Perez, Carmen Gavaldá Esteve. Dental management and respiratory disorders. $\mathbf{J}$ Clin Exp Dent. 2011; 3(3):e222-7. 